\title{
The readout system for the Fermilab Muon $g-2$ straw tracking detectors
}

\author{
James Mott*i \\ Boston University, Boston, MA 02215, U.S.A. \\ E-mail: jmottabu.edu
}

\begin{abstract}
The Fermilab Muon $g-2$ experiment aims to measure the anomalous magnetic moment of the muon to a precision of $140 \mathrm{ppb}$, a fourfold improvement over the previous E821 measurement at BNL. The measurement is extracted from an analysis of the modulation of the rate of higherenergy positrons from muon decays, detected by 24 calorimeters and 3 straw tracking detectors. The straw tracking detectors will be used to measure the beam profile, cross-calibrate the calorimeter, identify pile-up and track muons lost from the storage region. In addition, a tracker measurement of the vertical modulation of positrons will be used for a search for an electric dipole moment signal. Herein, the readout electronics of the straw tracker are described. Analogue signals from the straws are amplified, shaped and discriminated by custom ASDQ ASICs and the digital output is passed to an FPGA-based TDC which records the hit time with a resolution of $0.6 \mathrm{~ns}$. The hits are buffered and event records are created by FC7 and AMC13 modules in a MicroTCA crate, which are then passed into the MIDAS event-builder and data acquisition system.
\end{abstract}

38th International Conference on High Energy Physics

3-10 August 2016

Chicago, USA

\footnotetext{
* Speaker.

$\dagger$ On behalf of the Muon $g-2$ collaboration.
} 


\section{Muon $g-2$ Tracker}

The Fermilab Muon $g-2$ experimemt aims to measure the anomalous magnetic moment of the muon, $a_{\mu}=\frac{g-2}{2}$, to a precision of $140 \mathrm{ppb}$ [1]. An integral part of the experiment are the straw tracking detectors, which are required to measure the muon distribution. This is used for weighting magnetic field measurements and to calculate the two largest corrections to $a_{\mu}$. In addition, the detectors will be used to cross-check calorimeter position and momentum measurements, pile-up and gain correction algorithms and to search for vertical oscillations as a signal from an electric dipole moment.

Three straw trackers are placed around the storage ring and identify tracks from decay positrons. These tracks are extrapolated back to the point of tangency, thus providing a non-destructive measurement of the muon distribution. Each tracker comprises eight identical modules containing 128 straws in four layers (Fig. 1a). The eight modules are housed in the muon storage vacuum chamber and staggered radially towards the beam region to maximise the detector acceptance (Fig. 1b). Further details on the tracking modules are available in [1].
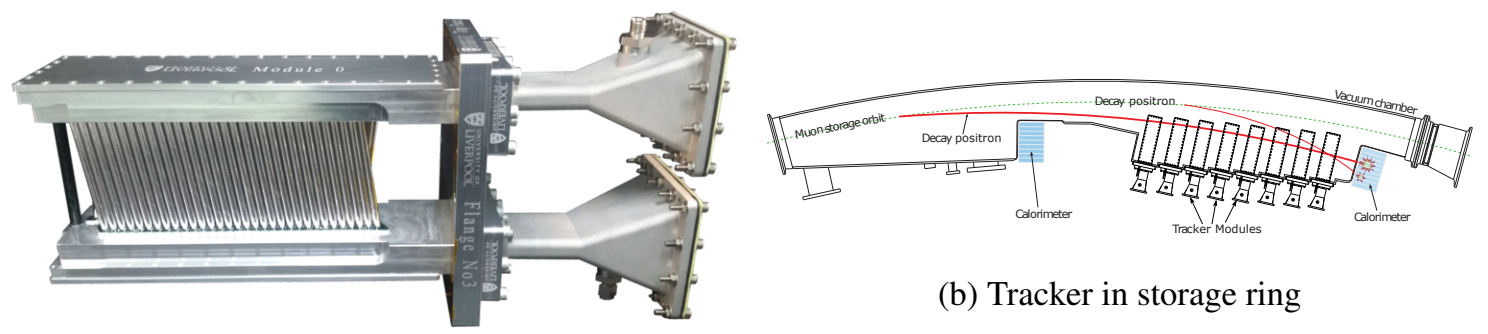

(b) Tracker in storage ring

(a) One tracker module

Figure 1: (a) A single tracker module with the first layer of 32 straws visible. (b) A plan view of a tracker inside one vacuum chamber of the storage ring.

\section{Frontend Readout Electronics}

The frontend readout electronics are those on each tracker module. Six different board types make up the readout chain as shown schematically in Figure 2. Each group of 16 straws is connected to an ASDQ board (Fig. 3a) housing two 8-channel ASDQ ASICs originally developed for the CDF Central Outer Tracker [2]. These chips preamplify, shape and discriminate signals on straw sense wires. Their specifications are a good match to our detector requirements, with fast charge collection ( $8 \mathrm{~ns})$, good double pulse resolution (30 ns), low power $(40 \mathrm{~mW} / \mathrm{ch})$ and low threshold ( $2 \mathrm{fC}$ ). Baseline restoration and ion tail compensation are also included.

The digital output of the ASDQ chips is transferred via flexible circuit boards, whose small thickness allows the necessary connections whilst maximising the vertical acceptance of the straws. These flexicables also pass power, reference voltages and test injection signals to the ASDQs. The cables connect to a feedthrough board (Fig. 3b), which acts as both a seal for the tracking gas volume and a backplane via which all other frontend boards communicate. The signals from two ASDQ boards (32 straws) are sent to one of two TDC boards (Fig. 3c), each of which houses two 


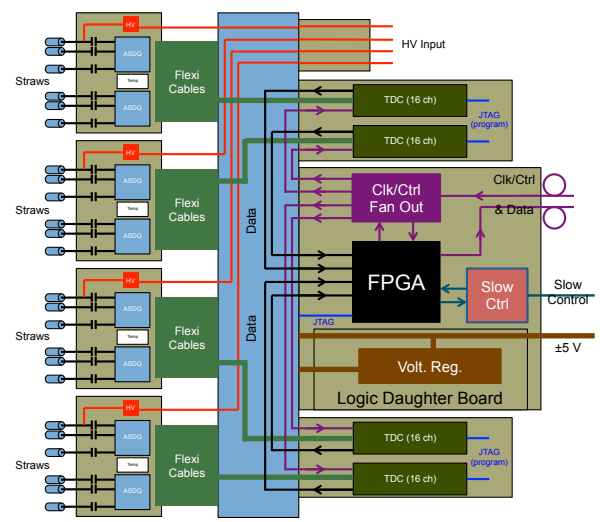

Figure 2: A schematic diagram of the frontend readout electronics for two straw layers. The signals proceed from the straws on the left, through the readout chain to the connections on the right.

Altera Cyclone III FPGAs. These digitise hit times to a precision of 625 ps LSB up to a total of 2016 hits per trigger.

Data from the four TDCs is transferred to a logic board (Fig. 3d), where a Xilinx Spartan-6 FPGA buffers, assembles and transmits events to the backend readout electronics over optical fibre. An external $10 \mathrm{MHz}$ reference clock with multiplexed trigger and control signals is sent to the logic board where it is multiplied and distributed to each of the four TDCs. In addition, the logic board regulates external power and supplies reference voltages to control the ASDQ chips. Both the TDC and logic board FPGAs can be remotely re-programmed.

In addition to the data-handling boards, an high voltage board accepts and filters four HV channels, before passing each to an ASDQ board via a separate cable. Further details on all boards can be found in [1].

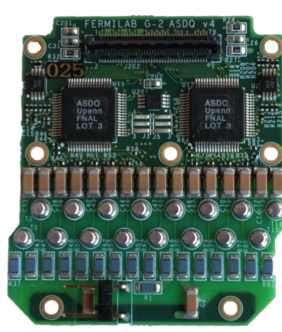

(a) ASDQ

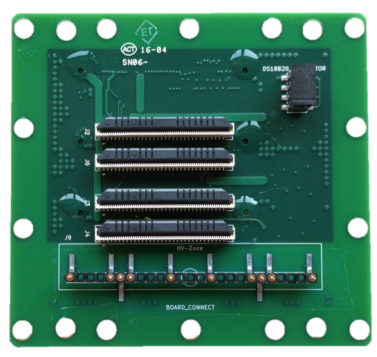

(b) Feedthrough

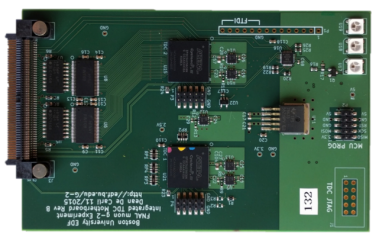

(c) TDC

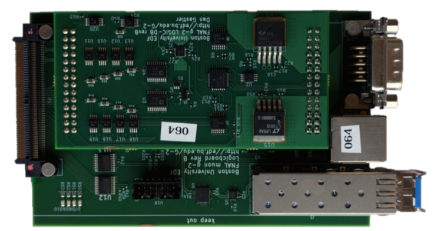

(d) Logic

Figure 3: Four of the different types of frontend electronics boards.

\section{Backend Readout Electronics}

The backend readout electronics are based on MicroTCA hardware developed for the CMS experiment. Optical fibres from one of the three trackers ( 8 modules, 16 logic boards, 1024 channels) are connected to an FC7 board. Three FC7 boards are connected to an AMC13 board [3] as in Figure 4. From the AMC13, data is passed to a DAQ PC running MIDAS. There are a total of 3072 straw channels and the required data rate is relatively low $(\sim 10 \mathrm{Mb} / \mathrm{s})$. 


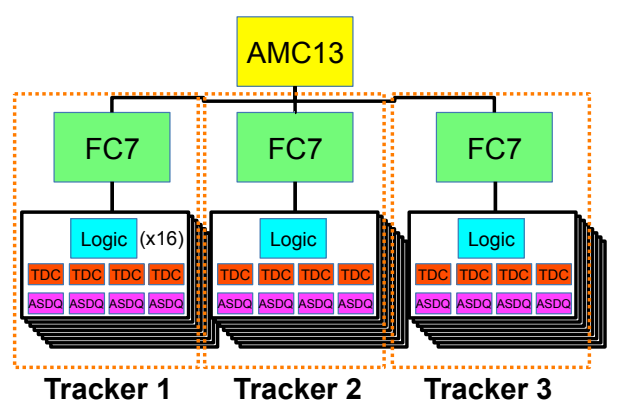

Figure 4: Backend Electronics Schematic

\section{Electronics and Straw Performance}

The performance of a tracker module and readout electronics was studied at Fermilab Test Beam Facility (FTBF) in 2015. All channels have satisfactory noise levels of $4 \mathrm{fC}$ or 25,000 $\mathrm{e}^{-}$ (Fig. 5a). Tracks from a silicon telescope were compared with those from the module under test and a resolution of $200 \mu \mathrm{m}$ was extracted (Fig. 5b). This is comfortably below the required individual hit position resolution of $300 \mu \mathrm{m}$.

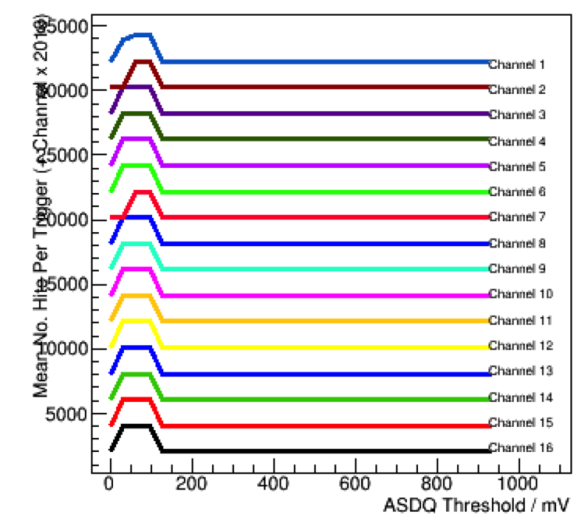

(a) No. hits vs. discriminator threshold

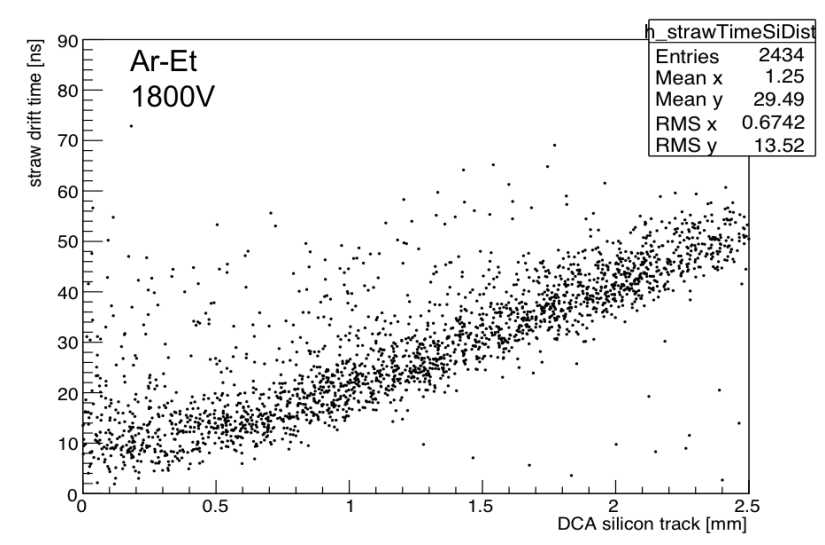

(b) Straw drift time vs. silicon track position

Figure 5: Performance of a tracker module at the FNAL Test Beam Facility (FTBF).

This work was supported in part by the US DOE.

\section{References}

[1] J. Grange et al., Muon (g-2) Technical Design Report, arXiv:1501.06858 [physics.ins-det]

[2] W. Bokhari et al., The ASDQ ASIC for the Front End electronics of the COT, CDF/DOC/Tracking/CDFR/4514 (1999)

[3] E. Hazen et al., The AMC13XG: a new generation clock/timing/DAQ module for CMS MicroTCA JINST 8 C12036 (2013) 